\title{
HOLOMORPHIC CONVEXITY OF TEICHMÜLLER SPACES ${ }^{1}$
}

\author{
BY LIPMAN BERS AND LEON EHRENPREIS
}

Communicated June 22, 1963

Let $B$ be the complex Banach space of holomorphic functions $\phi(z)=\phi(x+i y)$ defined for $y<0$, with norm $\|\phi\|=\sup \left|y^{2} \phi(z)\right|$. The universal Teichmüller space $T$ may be considered as a subset of $B$ defined as follows [2], [7]. $\phi \in B$ belongs to $T$ if and only if there is a quasiconformal selfmapping $w(z)$ of the $z$-plane which leaves 0 and 1 fixed and is, for $y<0$, a conformal mapping with Schwarzian derivative $\phi(z)$. If this is the case we say that $w$ belongs to $\phi . T$ is a bounded domain in $B$ containing the origin. The so-called Teichmüller metric (see below) is defined in $T$; it is topologically equivalent to the metric of $B$. Every boundary point of $T$ has infinite Teichmüller distance from the origin.

If $Q \subset T$, we denote by $h(Q)$ the hull of $Q$ with respect to continuous holomorphic functions in $T . \psi \in T$ belongs to $h(Q)$ if and only if there is no continuous holomorphic function $f$ in $T$ such that $|f(\psi)|>|f(\phi)|$ for all $\phi \in Q$.

THEOREM 1. If $Q \subset T$ is bounded in the Teichmiiller metric, so is $h(Q)$.

Proof. For $\phi \in T$ let $K(\phi)$ denote the smallest dilitation of a mapping $w$ belonging to $\phi$. The function $K(\phi)$ is well defined and $\log K(\phi)$ is the Teichmüller distance of $\phi$ to the origin.

For $\phi \in T$ and any three real numbers $a<b<c$ set $f_{a, b, c}(\phi)$ $=(w(b)-w(a)) /(w(c)-w(a))$ where $w$ is any mapping belonging to $\phi$. These functions are well defined and one verifies, using [3], that they are continuous and holomorphic in $T$.

Let $\phi \in T$ and $K(\phi) \leqq \alpha$. Then there is a $w$ belonging to $\phi$ with dilitation not exceeding $\alpha$. Let $\Gamma$ be the image of the real axis under $w$; this curve depends only on $\phi$. Set $\chi(\zeta)=w\left(w^{-1}(\zeta)^{*}\right)$ where the asterisk denotes complex conjugation. Then $\chi$ is a quasireflection about $\Gamma$, that is an orientation-reversing topological selfmapping of the plane which leaves every point of $\Gamma$ fixed, and the dilitation of $\chi$ is at most $\alpha^{2}$. By a theorem of Ahlfors [2] it follows that $\left|f_{a, b, c}(\phi)\right| \leqq \beta$ for all $a<b<c$, where $\beta$ depends only on $\alpha$.

Assume now that $\left|f_{a, b, c}(\phi)\right| \leqq \alpha$ for all $a<b<c$ and let $\Gamma$ be the image of the real axis under a mapping $w$ belonging to $\phi$. Again by

1 Work supported under Contract No. Nonr-285(46) with the Office of Naval Research. 
[2], there exists a quasireflection $\chi$ about $\Gamma$ of dilitation not exceeding $\beta$, where $\beta$ depends only on $\alpha$. Set $w_{1}(x+i y)=w(x+i y)$ for $y \leqq 0$ and $w_{1}(x+i y)=\chi(w(x-i y))$ for $y>0$. Then $w_{1}$ belongs to $\phi$ and the dilitation of $w_{1}$ is at most $\beta$.

We conclude that if $K \leqq \alpha<\infty$ on a set $Q \subset T$, then $K \leqq \alpha^{\prime}<\infty$ on $h(Q)$ where $\alpha^{\prime}$ depends only on $\alpha$. This proves the theorem.

Let $G$ be a Fuchsian group with the real axis as fixed line. Let $B(G)$ be the closed subspace of $B$ consisting of those elements for which $\phi(z) d z^{2}$ is invariant under $G$. The Teichmüller space $T(G)$ of quasiconformal deformations of $G$ may be identified with a domain in $B(G)$, namely the component of $T \cap B(G)$ containing the origin [6], [7], [8]. We note that $\operatorname{dim} B(G)<\infty$ if and only if $G$ is finitely generated and of the first kind.

If $Q \subset T(G)$ is bounded in the Teichmüller metric, the same is true of the hull $h_{G}(Q)$ of $Q$ with respect to continuous holomorphic functions in $T(G)$, for $h_{G}(Q) \subset h(Q)$. If $\operatorname{dim} B(G)<\infty$, this means that $T(G)$ is holomorphically convex in the sense of Cartan-Thullen; thus we obtain

THEOREM 2. Finite-dimensional Teichmïller spaces are domains of holomorphy.

This theorem applies, in particular, if $G$ is a hyperbolic group representing a closed Riemann surface of genus $g>1$. In this case $T(G)$ may be identified with the Teichmüller space $T_{g}$ of such Riemann surfaces, and Theorem 2 yields some information on period matrices.

Let $H_{\theta}$ denote the set of symmetric $(g \times g)$ matrices with positive definite imaginary parts (Siegel's generalized upper half-plane). There exists a natural holomorphic mapping $t \rightarrow Z(t)$ of $T_{g}$ into $H_{g}$ defined as follows. A point $t \in T_{g}$ may be considered as (the conformal equivalence class of) a closed Riemann surface $S(t)$ together with a "standard" system of generators $\left\{a_{1}, b_{1}, a_{2}, \cdots, b_{0}\right\}$ of the fundamental group of $S(t)$. This system of generators defines a canonical homology basis $\left\{\alpha_{1}, \beta_{1}, \cdots, \beta_{g}\right\}$ on $S(t)$. We set $Z(t)=\left(Z_{i j}\right)$, where $Z_{i j}$ is the period over $\beta_{j}$ of the Abelian differential of the first kind on $S(t)$ which has periods 1 over $\alpha_{i}$ and 0 over $\alpha_{k}, k \neq i$.

We denote the closure of $Z\left(T_{g}\right)$ in $H_{g}$ by $A_{g}$ and set $B_{g}=A_{g}-Z\left(T_{g}\right)$. By Baily's theorem [4], $A_{\theta}$ and $B_{g}$ are analytic sets.

Theorem 3. For $g>3$, every point $b \in B_{g}$ with $\operatorname{dim}_{b} B_{g}<3 g-4$ is a singular point of $A_{0}$. 
Proof. It is known [1], [5], [9] that $\operatorname{dim} T_{\theta}=3 g-3$ and that the points $t \in T_{g}$ such that $S(t)$ is hyperelliptic form a $(2 g-1)$-dimensional subvariety $T_{g}^{\prime}$. Also, the rank of $Z$ is $3 g-3$ at all points of $T_{g}-T_{g}^{\prime}$ and $2 g-1$ at points of $T_{g}^{\prime}$ (so that $A_{2}=H_{2}, A_{3}=H_{3}$ ). We denote by $C_{\theta}$ the closure of $Z\left(T_{g}^{\prime}\right)$ in $H_{g}$.

Let $g>3$ and assume that there is a $b \in B_{g}$ with $\operatorname{dim}_{b} B_{g}=q \leqq 3 g-5$ which is a regular (simple) point of $A_{g}$. Then $b \notin C_{\theta}$ and there exists a neighborhood $M$ of $b$ in $H_{g}$ such that $M$ does not meet $C_{g}, M \cap A_{g}$ is holomorphically homeomorphic to a simply-connected domain in a $(3 g-3)$-dimensional number space, and $\operatorname{dim}\left(M \cap B_{\theta}\right)=q$. Set $N=\left(M \cap A_{g}\right)-\left(M \cap B_{g}\right)$. Then $N$ is a simply-connected domain in $Z\left(T_{g}\right)$ and there exists a domain $N_{0} \subset T_{g}$ such that $Z \mid N_{0}$ is a homeomorphism onto $N$. There is a sequence $\left\{t_{j}\right\} \subset N_{0}$ such that $\lim Z\left(t_{j}\right)$ is $b$. This sequence diverges in $T_{a}$ so, by Theorem 2 , there is a holomorphic function $f$ in $T_{g}$ such that the sequence $\left\{f\left(t_{j}\right)\right\}$ is unbounded. But $f \mid N_{0}=F \circ\left(Z \mid N_{0}\right)$, where $F$ is holomorphic in $N$ and hence, by Hartogs' theorem, can be continued analytically over $M \cap A_{g}$. Thus the sequence $\left\{F\left(Z\left(t_{j}\right)\right)\right\}$ converges. Contradiction.

REMARK. It is very likely that $\operatorname{dim} B_{g}=3 g-5$ (though there seems to be no proof of this in the literature). If so, our result can be restated as

Theorem $3^{\prime}$. For $g>3$ the singular set of $A_{g}$ is $B_{g} \cup C_{g}$.

Note added in proof (September 20, 1964). Theorem $3^{\prime}$ is indeed valid since $\operatorname{dim} B_{g}=3 g-5$. More precisely, every element of $B_{o}$ is of the form $\beta(Z)$ where $Z$ is a direct sum of $k>1$ matrices $Z_{j} \in A_{g_{j}}$ with $g_{1}+\cdots+g_{k}=g$ and $\beta$ is an element of the group $\Gamma_{g}$ of holomorphic automorphisms of $H_{g}$ defined by integral symplectic matrices. A derivation of this statement from the results of Matsusaka and Hoyt (see [4] for references) has been communicated to us by Mumford. The key fact is that for a fixed integer $n>3$ the complex manifold $X=H_{g} / \Gamma_{g}(n)$, where $\Gamma_{g}(n)$ denotes the subgroup of $\Gamma_{g}$ corresponding to matrices congruent to the identity $\bmod n$, can be realized as a Zariski open subset of a projective algebraic variety, and that the canonical family of polarized Abelian varieties defined over $H_{\theta}$ is induced by an algebraic family defined over $X$.

We also note that the proof of Theorem 3 can be refined so as to give the following sharper result. Let $b$ be a point of $B_{g}$ but not of $C_{g}$, and let $M$ be a connected neighborhood of $b$ in $H_{g}$ which does not meet $C_{g}$. Then the intersection of $M$ with $A_{g}-B_{g}$ has an infinite fundamental group. 


\section{REFERENCES}

1. Lars Ahlfors, The complex analytic structure of the space of closed Riemann surfaces, pp. 45-66, Analytic functions, Princeton Univ. Press, Princeton, N. J., 1960.

2. - Quasiconformal reflections, Acta Math. 109 (1963), 291-301.

3. Lars Ahlfors and Lipman Bers, Riemann's mapping theorem for variable metrics, Ann. of Math. (2) 72 (1960), 385-404.

4. W. L. Baily, Jr., On the moduli of Jacobian varieties, Ann. of Math. (2) 71 (1960), 303-314.

5. Lipman Bers, Holomorphic differentials as functions of moduli, Bull. Amer. Math. Soc. 67 (1961), 206-210.

6. - Correction to "Spaces of Riemann surfaces as bounded domains," Bull. Amer. Math. Soc. 67 (1961), 465-466.

7. - A A - - A tomorphic forms and general Teichmüller spaces, Proc. Conf. Compl. Anal., Univ. of Minnesota, Minneapolis, Minn., Univ. of Minnesota Press, (to appear).

8. C. J. Earle, The Teichmiiller space of an arbitrary Fuchsian group, Bull. Amer. Math. Soc. 70 (1964), 699-701.

9. H. E. Rauch, On the transcendental moduli of algebraic Riemann surfaces, Proc. Nat. Acad. Sci. U.S.A. 41 (1955), 42-49.

NeW York UNIVERSITY 